\section{New Results on Stability of Discrete-Time Systems With Time-Varying State Delay}

Huijun Gao and Tongwen Chen

\begin{abstract}
This note is concerned with the stability analysis of discrete-time systems with time-varying state delay. By defining new Lyapunov functions and by making use of novel techniques to achieve delay dependence, several new conditions are obtained for the asymptotic stability of these systems. The merit of the proposed conditions lies in their less conservativeness, which is achieved by circumventing the utilization of some bounding inequalities for cross products between two vectors and by paying careful attention to the subtle difference between the terms $\sum_{m=k-d_{k}}^{k-1}(\bullet)$ and $\sum_{m=k-d_{M}}^{k-1}(\bullet)$, which is largely ignored in the existing literature. These conditions are shown, via several examples, to be much less conservative than some existing result.
\end{abstract}

Index Terms-Delay dependence, networked control systems, robust stability, time-delay systems.

\section{INTRODUCTION}

Over the past few decades, time-delay systems have drawn much attention from researchers throughout the world. This is due to their important role in many practical systems. A great number of research results concerning time-delay systems exist in the literature (see [1], [3]-[6], [8]-[15], [17], and the references therein). The stability of time-delay systems has been well known to be a fundamental problem because of its importance in the analysis and synthesis of such systems.

Discrete-time systems with state delay have strong background in engineering applications, among which network based control has been well recognized to be a typical example. If the delay is constant, one can transform a delayed system into a delay-free one by using state augmentation techniques. In this way, stability of such systems can be readily tested by employing classical results on stability analysis. Such an approach, however, is not always implementable as the dimension of the augmented system increases with the delay size. That is, when the delay is large, the augmented system will become much complex and thus difficult to analyze and synthesize. Moreover, the state augmentation technique is usually not applicable to the time-varying delay case, which is more frequently encountered than the constant delay case in practice. The reason is that for time-varying delay systems, the transformed systems usually have time-varying matrix coefficients, which are apparently difficult to analyze using available tools. Consequently, much effort has been made towards investigating the stability of discrete time-delay systems via Lyapunov approaches [5]-[7], [18]. However, it is worth mentioning that most of the results are concerned with the constant delay case, and according to the best of the authors' knowledge, little progress has been reported for the stability analysis of discrete-time systems with time-varying state delay, which motivates the present study.

Manuscript received April 10, 2006; revised September 27, 2006. Recommended by Associate Editor A. Hansson. This work was supported by an Alberta Ingenuity Fellowship, a Killam Postdoctoral Fellowship, Natural Sciences and Engineering Research Council of Canada, National Natural Science Foundation of China under Grants 60528007 and 60504008 , and by the Program for New Century Excellent Talents in Universities, China.

H. Gao is with Harbin Institute of Technology, Harbin 150001, China. He is now visiting the Department of Electrical and Computer Engineering, University of Alberta, Edmonton, AB T6G 2V4, Canada (e-mail: hjgao@hit.edu.cn).

T. Chen is with the Department of Electrical and Computer Engineering, University of Alberta, Edmonton, AB T6G 2V4, Canada (e-mail: tchen@ece.ualberta.ca).

Digital Object Identifier 10.1109/TAC.2006.890320
In this note, we revisit the problem of stability analysis for discrete-time systems with a time-varying delay in the state, which has been investigated in [7]. By defining new Lyapunov functions, and by making use of novel techniques to achieve delay dependence, several new results are presented for the asymptotic stability. The proposed conditions are advantageous in terms of less conservativeness, which is achieved by circumventing the utilization of bounding inequalities for cross products between two vectors and by paying careful attention to the subtle difference between the terms $\sum_{m=k-d_{k}}^{k-1}(\bullet)$ and $\sum_{m=k-d_{M}}^{k-1}(\bullet)$, which is largely ignored in the previous results. Several examples, including an inverted pendulum system with delayed input, are provided to illustrate the advantage of the developed results.

Notation: $\mathbb{R}^{n}$ denotes the $n$-dimensional Euclidean space and the notation $P>0(\geq 0)$ means that $P$ is real symmetric and positive definite (semidefinite). In symmetric block matrices or complex matrix expressions, we use an asterisk $(*)$ to represent a term that is induced by symmetry and $\operatorname{diag}\{\ldots\}$ stands for a block-diagonal matrix. Matrices, if their dimensions are not explicitly stated, are assumed to be compatible for algebraic operations.

\section{MAIN Results}

\section{A. New Stability Condition}

Consider the following discrete-time system $\mathcal{S}$ with a time-varying delay in the state:

$$
\begin{aligned}
\mathcal{S}: \quad x(k+1) & =A x(k)+B x\left(k-d_{k}\right) \\
x(k) & =\phi(k) \quad k=-d_{M},-d_{M}+1, \ldots, 0 .
\end{aligned}
$$

Here, $x(k) \in \mathbb{R}^{n}$ is the state vector; $A, A_{d}$ are system matrices with compatible dimensions; $d_{k}$ is a time-varying delay in the state. A natural assumption on $d_{k}$ is made as follows.

Assumption 1: The time delays $d_{k}$ are assumed to be time-varying and satisfy $d_{m} \leq d_{k} \leq d_{M}$, where $d_{m}$ and $d_{M}$ are constant positive scalars representing the lower and upper delays, respectively.

In system $\mathcal{S},\left\{\phi(k), k=-d_{M},-d_{M}+1, \ldots, 0\right\}$ is a given initial condition sequence.

Remark 1: The assumption on the time delay $d_{k}$ in Assumption 1 characterizes the real situation in many practical applications. A typical example containing time delays that can be characterized by Assumption 1 can be found in networked control systems, where the delays induced by the network transmission (either from sensor to controller or from controller to actuator) are actually time-varying, and can be assumed to have lower and upper delay bounds without loss of generality.

A stability condition for system $\mathcal{S}$ in (1) with Assumption 1 has been proposed in [7], by defining the following Lyapunov function:

$$
\begin{aligned}
V(k) & =V_{1}(k)+V_{2}(k)+V_{3}(k)+V_{4}(k) \\
V_{1}(k) & =x^{T}(k) P x(k) \quad V_{2}(k)=\sum_{i=k-d_{k}}^{k-1} x^{T}(i) Q x(k) \\
V_{3}(k) & =\sum_{j=-d_{M}+2}^{-d_{m}+1} \sum_{i=k+j-1}^{k-1} x^{T}(i) Q x(i) \\
V_{4}(k) & =\sum_{i=-d_{M}}^{-1} \sum_{m=k+i}^{k-1} \eta^{T}(m) Z \eta(m) \\
\eta(k) & =x(k+1)-x(k) .
\end{aligned}
$$

The calculation of the forward difference $\Delta V(k)=V(k+1)-V(k)$, based on which the stability condition was derived, deserves some 
comments. Firstly, to bring the information of the delay size into the final result (to achieve delay dependence), a model transformation was performed to the original system $\mathcal{S}$ in (1) and, thus, an inequality was inevitably employed to bound the inner product between two vectors, which gave rise to possible conservativeness. Second, the calculation of $\Delta V_{1}(k)$ and $\Delta V_{4}(k)$ led to $\sum_{m=k-d_{k}}^{k-1} \eta^{T}(m) Z \eta(m)$ and $-\sum_{m=k-d_{M}}^{k-1} \eta^{T}(m) Z \eta(m)$, respectively. In order to achieve counteraction, $\sum_{m=k-d_{k}}^{k-1} \eta^{T}(m) Z \eta(m)$ was enlarged to $\sum_{m=k-d_{M}}^{k-1} \eta^{T}(m) Z \eta(m)$. In other words, $\sum_{m=k-d_{M}}^{k-d_{k}-1} \eta^{T}(m) Z \eta(m)$ was additionally introduced, which was the second source of possible conservativeness. In what follows, we present a new stability condition for the discrete-time system $\mathcal{S}$ in (1) by seeking improvement from the above two aspects.

Theorem 1: System $\mathcal{S}$ in (1) with Assumption 1 is asymptotically stable if there exist matrices $P>0, Q \geq 0, R \geq 0, Z_{i}>0, i=$ $1,2, M, S, N$ satisfying

$$
\left[\begin{array}{cc}
\Xi_{1}+\Xi_{2}+\Xi_{2}^{T}+\Xi_{3} & \Xi_{4} \\
* & \Xi_{5}
\end{array}\right]<0
$$

where

$$
\begin{aligned}
& \Xi_{1}=\left[\begin{array}{ccc}
A^{T} P A-P+\tau Q+R & A^{T} P B & 0 \\
* & B^{T} P B-Q & 0 \\
* & * & -R
\end{array}\right] \\
& \tau=\left(d_{M}-d_{m}+1\right) \\
& \Xi_{2}=\left[\begin{array}{lll}
M+N & S-M & -S-N
\end{array}\right] \\
& \Xi_{3}=d_{M}\left[\begin{array}{lll}
A-I & B & 0
\end{array}\right]^{T}\left(Z_{1}+Z_{2}\right)[A-I \quad B \quad 0] \\
& \Xi_{4}=\left[\begin{array}{lll}
\sqrt{d_{M}} M & \sqrt{d_{M}-d_{m}} S & \sqrt{d_{M}} N
\end{array}\right] \\
& \Xi_{5}=\operatorname{diag}\left\{-Z_{1},-Z_{1},-Z_{2}\right\} \text {. }
\end{aligned}
$$

Proof: To prove the theorem, choose a Lyapunov function candidate as

$$
\begin{aligned}
& V(k)=V_{1}(k)+V_{2}(k)+V_{3}(k)+V_{4}(k)+V_{5}(k) \\
& V_{1}(k)=x^{T}(k) P x(k) \quad V_{2}(k)=\sum_{i=k-d_{k}}^{k-1} x^{T}(i) Q x(i) \\
& V_{3}(k)=\sum_{i=k-d_{M}}^{k-1} x^{T}(i) R x(i) \\
& V_{4}(k)=\sum_{j=-d_{M}+1}^{-d_{m}} \sum_{i=k+j}^{k-1} x^{T}(i) Q x(i) \\
& V_{5}(k)=\sum_{i=-d_{M}}^{-1} \sum_{m=k+i}^{k-1} \eta^{T}(m)\left(Z_{1}+Z_{2}\right) \eta(m) \\
& \eta(k)=x(k+1)-x(k)
\end{aligned}
$$

where $P>0, Q \geq 0, R \geq 0, Z_{i}>0, i=1,2$, are matrices to be determined. Define $\Delta V(k)=V(k+1)-V(k)$, then along the solution of (1) we have

$$
\begin{aligned}
\Delta V_{1}(k)= & x^{T}(k+1) P x(k+1)-x^{T}(k) \operatorname{Px}(k) \\
\Delta V_{2}(k) \leq & x^{T}(k) Q x(k)-x^{T}\left(k-d_{k}\right) Q x\left(k-d_{k}\right) \\
& +\sum_{i=k-d_{M}+1}^{k-d_{m}} x^{T}(i) Q x(i) \\
\Delta V_{3}(k)= & x^{T}(k) R x(k)-x^{T}\left(k-d_{M}\right) R x\left(k-d_{M}\right) \\
\Delta V_{4}(k)= & \left(d_{M}-d_{m}\right) x^{T}(k) Q x(k)
\end{aligned}
$$

$$
\begin{aligned}
& -\sum_{i=k-d_{M}+1}^{k-d_{m}} x^{T}(i) Q x(i) \\
\Delta V_{5}(k)= & \sum_{i=-d_{M}}^{-1}\left[\eta^{T}(k)\left(Z_{1}+Z_{2}\right) \eta(k)\right. \\
& \left.-\eta^{T}(k+i)\left(Z_{1}+Z_{2}\right) \eta(k+i)\right] \\
= & d_{M} \eta^{T}(k)\left(Z_{1}+Z_{2}\right) \eta(k)-\sum_{l=k-d_{k}}^{k-1} \eta^{T}(l) Z_{1} \eta(l) \\
& -\sum_{l=k-d_{M}}^{k-d_{k}-1} \eta^{T}(l) Z_{1} \eta(l)-\sum_{l=k-d_{M}}^{k-1} \eta^{T}(l) Z_{2} \eta(l) .
\end{aligned}
$$

Define $\zeta(k)=\left[x^{T}(k) x^{T}\left(k-d_{k}\right) x^{T}\left(k-d_{M}\right)\right]^{T}$. Then, we have

$$
\begin{aligned}
& \Delta V(k) \leq x^{T}(k)\left[A x(k)+B x\left(k-d_{k}\right)\right]^{T} P[A x(k) \\
& \left.+B x\left(k-d_{k}\right)\right] x(k)-x^{T}(k) P x(k) \\
& +\left(d_{M}-d_{m}+1\right) x^{T}(k) Q x(k) \\
& -x^{T}\left(k-d_{k}\right) Q x\left(k-d_{k}\right) \\
& +x^{T}(k) R x(k)-x^{T}\left(k-d_{M}\right) R x\left(k-d_{M}\right) \\
& +d_{M}\left[(A-I) x(k)+B x\left(k-d_{k}\right)\right]^{T} \\
& \times Z\left[(A-I) x(k)+B x\left(k-d_{k}\right)\right] \\
& -\sum_{l=k-d_{k}}^{k-1} \eta^{T}(l) Z_{1} \eta(l) \\
& -\sum_{l=k-d_{M}}^{k-d_{k}-1} \eta^{T}(l) Z_{1} \eta(l) \\
& -\sum_{l=k-d_{M}}^{k-1} \eta^{T}(l) Z_{2} \eta(l) \\
& +2 \zeta^{T}(k) M\left[x(k)-x\left(k-d_{k}\right)-\sum_{l=k-d_{k}}^{k-1} \eta(l)\right] \\
& +2 \zeta^{T}(k) S\left[x\left(k-d_{k}\right)-x\left(k-d_{M}\right)\right. \\
& \left.-\sum_{l=k-d_{M}}^{k-d_{k}-1} \eta(l)\right] \\
& +2 \zeta^{T}(k) N\left[x(k)-x\left(k-d_{M}\right)-\sum_{l=k-d_{M}}^{k-1} \eta(l)\right] \\
& \leq \zeta^{T}(k)\left[\Xi_{1}+\Xi_{2}+\Xi_{2}^{T}+\Xi_{3}+d_{M} M Z_{1}^{-1} M^{T}\right. \\
& \left.+\left(d_{M}-d_{m}\right) S Z_{1}^{-1} S^{T}+d_{M} N Z_{2}^{-1} N^{T}\right] \zeta(k) \\
& -\sum_{l=k-d_{k}}^{k-1}\left[\zeta^{T}(k) M+\eta^{T}(l) Z_{1}\right] Z_{1}^{-1}\left[M^{T} \zeta(k)\right. \\
& \left.+Z_{1} \eta(l)\right] \\
& -\sum_{l=k-d_{M}}^{k-d_{k}-1}\left[\zeta^{T}(k) S+\eta^{T}(l) Z_{1}\right] Z_{1}^{-1} \\
& \times\left[S^{T} \zeta(k)+Z_{1} \eta(l)\right] \\
& -\sum_{l=k-d_{M}}^{k-1}\left[\zeta^{T}(k) N+\eta^{T}(l) Z_{2}\right] Z_{2}^{-1}\left[N^{T} \zeta(k)\right. \\
& \left.+Z_{2} \eta(l)\right] \text {. }
\end{aligned}
$$


Since $Z_{i}>0, i=1,2$, the last three terms are all nonpositive. By the Schur complement, (2) guarantees $\Xi_{1}+\Xi_{2}+\Xi_{2}^{T}+\Xi_{3}+$ $d_{M} M Z_{1}^{-1} M^{T}+\left(d_{M}-d_{m}\right) S Z_{1}^{-1} S^{T}+d_{M} N Z_{2}^{-1} N^{T}<0$. Therefore, from (6) we have $\Delta V(k)<-\epsilon\|x(k)\|^{2}$ for a sufficiently small $\epsilon>0$ and $x(k) \neq 0$, and the asymptotic stability is established.

Remark 2: From the proof of Theorem 1, we can see that to achieve delay dependence, no model transformation has been performed to the original system and thus no bounding technique has been employed to seek upper bounds of the inner product between two vectors. This corresponds to the first comment preceding Theorem 1. Moreover, when calculating the forward difference of $\Delta V_{5}(k)$, we get $-\sum_{l=k-d_{M}}^{k-1} \eta^{T}(l) Z_{1} \eta(l)$. In order to reduce possible conservativeness, we have not enlarged this to $-\sum_{l=k-d_{k}}^{k-1} \eta^{T}(l) Z_{1} \eta(l)$. Instead, we have separated it into two parts, that is, $-\sum_{l=k-d_{k}}^{k-1} \eta^{T}(l) Z_{1} \eta(l)$ and $-\sum_{l=k-d_{M}}^{k-d_{k}-1} \eta^{T}(l) Z_{1} \eta(l)$, and treated them using different free-weighting techniques, respectively. As can be seen in the examples given later, these ideas render the stability condition presented in Theorem 1 to be much less conservative than that in [7].

\section{B. Robust Stability Condition}

The stability condition developed in the above subsection can be further extended to cope with uncertain systems. In this section, we assume that the matrices in the dynamic equation of $\mathcal{S}$ in (1) have norm bounded uncertainty [16] described by the following assumption.

Assumption 2: Assume that the matrices $A, B$ in the dynamic equation of $\mathcal{S}$ in (1) have the following form:

$$
A=A_{0}+\Delta A \quad B=B_{0}+\Delta B
$$

where $A_{0}, B_{0}$ are known constant matrices of appropriate dimensions. $\Delta A, \Delta B$ are real-valued time-varying matrix functions representing norm-bounded parameter uncertainties satisfying

$$
\left[\begin{array}{ll}
\Delta A & \Delta B
\end{array}\right]=G \Delta(k)\left[\begin{array}{ll}
H_{1} & H_{2}
\end{array}\right]
$$

where $\Delta(k)$ is a real uncertain matrix function with Lebesgue measurable elements satisfying $\Delta^{T}(k) \Delta(k) \leq I$, and $G, H_{1}, H_{2}$ are known real constant matrices of appropriate dimensions. These matrices specify how the uncertain parameters in $\Delta(k)$ enter the nominal matrices $A_{0}, B_{0}$.

Then, extension of Theorem 1 is presented in the following corollary (The proof follows similar lines as in [7]).
Corollary 2: System $\mathcal{S}$ in (1) with Assumptions 1 and 2 is robustly asymptotically stable if there exist matrices $P>0, Q \geq 0, R \geq$ $0, Z_{i}>0, i=1,2, M, S, N$ and scalar $\epsilon>0$ satisfying (8), as shown at the bottom of the page, where $\tau, \Xi_{i}, i=2,4,5$, are given in (3) and the second equation shown at the bottom of the page holds.

\section{Augmented Stability Condition}

In the following, we present another new stability result.

Theorem 3: System $\mathcal{S}$ in (1) with Assumption 1 is asymptotically stable if there exist matrices

$$
\begin{aligned}
& P=\left[\begin{array}{ccc}
P_{11} & P_{12} & P_{13} \\
* & P_{22} & P_{23} \\
* & * & P_{33}
\end{array}\right]>0, Q=\left[\begin{array}{cc}
Q_{11} & Q_{12} \\
* & Q_{22}
\end{array}\right] \geq 0 \\
& R=\left[\begin{array}{cc}
R_{11} & R_{12} \\
* & R_{22}
\end{array}\right] \geq 0
\end{aligned}
$$

and $Z_{i}>0, i=1,2, M, S, N, T$ satisfying

$$
\left[\begin{array}{cc}
\Upsilon_{1}+\Upsilon_{2}+\Upsilon_{2}^{T}+\Upsilon_{3}+\Upsilon_{3}^{T} & \Xi_{4} \\
* & \Xi_{5}
\end{array}\right]<0
$$

where $\tau, \Xi_{4}, \Xi_{5}$, are given in (3) and

$$
\begin{aligned}
\Upsilon_{1} & =\left[\begin{array}{cccccc}
\Upsilon_{4} & 0 & 0 & \Upsilon_{5} & P_{12} & P_{13} \\
* & -Q_{11} & 0 & P_{12}^{T} & P_{22}-Q_{12} & P_{23} \\
* & * & -R_{11} & P_{13}^{T} & P_{23}^{T} & P_{33}-R_{12} \\
* & * & * & \Upsilon_{6} & P_{12} & P_{13} \\
* & * & * & * & P_{22}-Q_{22} & P_{23} \\
* & * & * & * & * & P_{33}-R_{22}
\end{array}\right] \\
\Upsilon_{2} & =\left[\begin{array}{llllll}
M+N & S-M & -S-N & 0 & 0 & 0
\end{array}\right] \\
\Upsilon_{4} & =\tau Q_{11}+R_{11} \\
\Upsilon_{3} & =T[-(A-I) \\
\Upsilon_{5} & =P_{11}+\tau Q_{12}+R_{12} \\
\Upsilon_{6} & =P_{11}+\tau Q_{22}+R_{22}+d_{M}\left(Z_{1}+Z_{2}\right) .
\end{aligned}
$$

Proof: To prove the theorem, choose a Lyapunov function candidate as

$$
\begin{gathered}
V(k)=V_{1}(k)+V_{2}(k)+V_{3}(k)+V_{4}(k)+V_{5}(k) \\
V_{1}(k)=\zeta^{T}(k) P \zeta(k) \quad \zeta(k)=\left[\begin{array}{c}
x(k) \\
x\left(k-d_{k}\right) \\
x\left(k-d_{M}\right)
\end{array}\right]
\end{gathered}
$$

$$
\left[\begin{array}{ccccc}
-\left(Z_{1}+Z_{2}\right) & 0 & \Phi_{4} & 0 & \sqrt{d_{M}}\left(Z_{1}+Z_{2}\right) G \\
* & -P & \Phi_{3} & 0 & P G \\
* & * & \Phi_{1}+\Xi_{2}+\Xi_{2}^{T}+\Phi_{2} & \Xi_{4} & 0 \\
* & * & * & \Xi_{5} & 0 \\
* & * & * & * & -\epsilon I
\end{array}\right]<0
$$

$$
\begin{aligned}
& \Phi_{1}=\left[\begin{array}{ccc}
-P+\tau Q+R & 0 & 0 \\
* & -Q & 0 \\
* & * & -R
\end{array}\right] \\
& \Phi_{2}=\epsilon\left[\begin{array}{lll}
H_{1} & H_{2} & 0
\end{array}\right]^{T}\left[\begin{array}{lll}
H_{1} & H_{2} & 0
\end{array}\right] \\
& \Phi_{3}=\left[\begin{array}{lll}
P A_{0} & P B_{0} & 0
\end{array}\right] \quad \Phi_{4}=\left[\sqrt{d_{M}}\left(Z_{1}+Z_{2}\right)\left(A_{0}-I\right) \quad \sqrt{d_{M}}\left(Z_{1}+Z_{2}\right) B_{0} \quad 0\right]
\end{aligned}
$$




$$
\begin{aligned}
V_{2}(k) & =\sum_{i=k-d_{k}}^{k-1} \lambda^{T}(i) Q \lambda(i) \\
V_{3}(k) & =\sum_{i=k-d_{M}}^{k-1} \lambda^{T}(i) R \lambda(i) \\
V_{4}(k) & =\sum_{j=-d_{M}+1}^{-d_{m}} \sum_{i=k+j}^{k-1} \lambda^{T}(i) Q \lambda(i) \quad \lambda(k)=\left[\begin{array}{c}
x(k) \\
\eta(k)
\end{array}\right] \\
V_{5}(k) & =\sum_{i=-d_{M}}^{-1} \sum_{m=k+i}^{k-1} \eta^{T}(m)\left(Z_{1}+Z_{2}\right) \eta(m) \\
\eta(k) & =x(k+1)-x(k)
\end{aligned}
$$

where $P, Q, R$ with structures given in (9), and $Z_{i}>0, i=1,2$, are matrices to be determined. Define $\Delta V(k)=V(k+1)-V(k)$. Then, along the solution of (1) we have

$$
\begin{aligned}
\Delta V_{1}(k)=2\left[\begin{array}{c}
x(k) \\
x\left(k-d_{k}\right) \\
x\left(k-d_{M}\right)
\end{array}\right]^{T} P\left[\begin{array}{c}
\eta(k) \\
\eta\left(k-d_{k}\right) \\
\eta\left(k-d_{M}\right)
\end{array}\right] \\
+\left[\begin{array}{c}
\eta(k) \\
\eta\left(k-d_{k}\right) \\
\eta\left(k-d_{M}\right)
\end{array}\right]^{T} P\left[\begin{array}{c}
\eta(k) \\
\eta\left(k-d_{k}\right) \\
\eta\left(k-d_{M}\right)
\end{array}\right] .
\end{aligned}
$$

By following similar lines as in the proof of Theorem 1, we obtain

$$
\begin{aligned}
& \Delta V_{2}(k) \\
& \leq \lambda^{T}(k) Q \lambda(k)-\lambda^{T}\left(k-d_{k}\right) Q \lambda\left(k-d_{k}\right) \\
& \quad+\sum_{i=k-d_{M}+1}^{k-d_{m}} \lambda^{T}(i) Q \lambda(i) \\
& \Delta V_{3}(k) \\
& =\lambda^{T}(k) R \lambda(k)-\lambda^{T}\left(k-d_{M}\right) R \lambda\left(k-d_{M}\right) \\
& \Delta V_{4}(k) \\
& =\left(d_{M}-d_{m}\right) \lambda^{T}(k) Q \lambda(k)-\sum_{i=k-d_{M}+1}^{k-d_{m}} \lambda^{T}(i) Q \lambda(i) \\
& \Delta V_{5}(k) \\
& =d_{M} \eta^{T}(k)\left(Z_{1}+Z_{2}\right) \eta(k)-\sum_{l=k-d_{k}}^{k-1} \eta^{T}(l) Z_{1} \eta(l) \\
& \quad-\sum_{l=k-d_{M}}^{k-d_{k}-1} \eta^{T}(l) Z_{1} \eta(l)-\sum_{l=k-d_{M}}^{k-1} \eta^{T}(l) Z_{2} \eta(l) .
\end{aligned}
$$

In addition, based on (1), we have

$$
\eta(k)=x(k+1)-x(k)=(A-I) x(k)+B x\left(k-d_{k}\right) .
$$

Then, for any matrix $T$, we have

$$
\nu^{T}(k) T\left[\eta(k)-(A-I) x(k)-B x\left(k-d_{k}\right)\right]=0 .
$$

Define $\nu(k)=\left[x^{T}(k) x^{T}\left(k-d_{k}\right) x^{T}\left(k-d_{M}\right)\right.$ eta $a^{T}(k) \eta^{T}(k-$ $\left.\left.d_{k}\right) \eta^{T}\left(k-d_{M}\right)\right]^{T}$. Then, similarly to (6), we have

$$
\begin{aligned}
\leq & \nu^{T}(k)\left[\Upsilon_{1}+\Upsilon_{2}+\Upsilon_{2}^{T}+\Upsilon_{3}+d_{M} M Z_{1}^{-1} M^{T}\right. \\
& \left.+\left(d_{M}-d_{m}\right) S Z_{1}^{-1} S^{T}+d_{M} N Z_{2}^{-1} N^{T}\right] \nu(k) \\
- & \sum_{l=k-d_{k}}^{k-1}\left[v^{T}(k) M+\eta^{T}(l) Z_{1}\right] Z_{1}^{-1} \\
& \times\left[M^{T} v(k)+Z_{1} \eta(l)\right] \\
- & \sum_{l=k-d_{M}}^{k-d_{k}-1}\left[v^{T}(k) S+\eta^{T}(l) Z_{1}\right] Z_{1}^{-1} \\
& \times\left[S^{T} v(k)+Z_{1} \eta(l)\right] \\
& -\sum_{l=k-d_{M}}^{k-1}\left[v^{T}(k) N+\eta^{T}(l) Z_{2}\right] Z_{2}^{-1} \\
& \times\left[N^{T} v(k)+Z_{2} \eta(l)\right] .
\end{aligned}
$$

Since $Z_{i}>0, i=1,2$, the last three terms in the previous equation are all nonpositive. By the Schur complement, (10) guarantees $\Upsilon_{1}+\Upsilon_{2}+$ $\Upsilon_{2}^{T}+\Upsilon_{3}+d_{M} M Z_{1}^{-1} M^{T}+\left(d_{M}-d_{m}\right) S Z_{1}^{-1} S^{T}+d_{M} N Z_{2}^{-1} N^{T}<$ 0 . Therefore, from (20) we have $\Delta V(k)<-\epsilon\|x(k)\|^{2}$ for a sufficiently small $\epsilon>0$ and $x(k) \neq 0$, and the asymptotic stability is established.

Remark 3: By introducing more matrix variables, Theorem 3 gives another stability condition. One will naturally ask whether it can be estabilished theoretically that Theorem 3 is generally less conservative than Theorem 1. To answer this question, let us select the following special structures for the matrix variables in Theorem 3:

$$
\begin{aligned}
P & =\left[\begin{array}{ccc}
P_{11} & 0 & 0 \\
* & \epsilon I & 0 \\
* & * & \epsilon I
\end{array}\right]>0 \quad Q=\left[\begin{array}{cc}
Q_{11} & 0 \\
* & 0
\end{array}\right] \geq 0 \\
R & =\left[\begin{array}{cc}
R_{11} & 0 \\
* & 0
\end{array}\right] \geq 0 \\
M & =\left[\begin{array}{c}
M_{1} \\
M_{2} \\
M_{3} \\
0 \\
0 \\
0
\end{array}\right] \quad S=\left[\begin{array}{c}
S_{1} \\
S_{2} \\
S_{3} \\
0 \\
0 \\
0
\end{array}\right] \quad N=\left[\begin{array}{c}
N_{1} \\
N_{2} \\
N_{3} \\
0 \\
0 \\
0
\end{array}\right] \quad T=\left[\begin{array}{c}
T_{1} \\
T_{2} \\
T_{3} \\
T_{4} \\
T_{5} \\
T_{6}
\end{array}\right]
\end{aligned}
$$

with $\epsilon$ being a sufficiently small positive constant. Then, under the aforementioned constraints, condition (10) can be written as

$$
W+X+X^{T}+U T V+(U T V)^{T}<0
$$

where the equation shown at the bottom of the next page holds. Then, by using the elimination lemma with respect to the matrix $T$, we can conclude that (21) is equivalent to (2). This means that by imposing special structures on the matrix variables in Theorem 3 , we readily obtain Theorem 1. In other words, Theorem 3 is generally less conservative than Theorem 1.

It is also worth noting that though Theorem 3 has the advantage of providing less conservative stability test, the matrix variables to be determined are much more than those in Theorem 1. In particular, in Theorem 3, the system matrices are involved in the products with six matrix variables $\left.\left(T_{1}, \ldots, T_{6}\right)\right)$, while in Theorem 1 only three matrix variables $\left(P, Z_{1}\right.$ and $\left.Z_{2}\right)$ are involved. Therefore, it is antipicated that Theorem 1 
TABLE I

CALCUlated UpPer Delay Bounds For DifFerent CASES

\begin{tabular}{c|c|c|c|c|c}
\hline & \multicolumn{5}{|c}{ Calculated $d_{M}$ for given $d_{m}$} \\
\hline & $d_{m}=2$ & $d_{m}=4$ & $d_{m}=6$ & $d_{m}=10$ & $d_{m}=12$ \\
\hline By [7] & 7 & 8 & 9 & 12 & 13 \\
\hline By Theorem 1 & 13 & 13 & 14 & 15 & 16 \\
\hline By Theorem 3 & 13 & 13 & 14 & 15 & 17 \\
\hline
\end{tabular}

is more powerful when used for synthesis problems, such as controller and filter designs, which is worth further researching.

\section{ILLUSTRATIVE EXAMPLES}

In this section, three examples are provided to illustrate the advantage of the proposed stability results.

Example 1: Consider the following discrete-time system with a time-varying state delay [7]:

$$
x(k+1)=\left[\begin{array}{cc}
0.8 & 0 \\
0.05 & 0.9
\end{array}\right] x(k)+\left[\begin{array}{cc}
-0.1 & 0 \\
-0.2 & -0.1
\end{array}\right] x\left(k-d_{k}\right) .
$$

Here, $d_{k}$ represents a time-varying state delay. Now assume the lower delay bound of $d_{k}$ is $d_{m}=2$, and we are interested in the upper delay bound $d_{M}$ below which the above system is asymptotically stable for all $d_{m} \leq d_{k} \leq d_{M}$. By using [7, Th. 1], it is found that the upper delay bound $d_{M}=7$. However, by applying Theorem 1, we obtain the upper delay bound $d_{M}=13$. Theorem 3 also yields $d_{M}=13$. A more detailed comparison is given in Table 1, from which we can see that the stability conditions presented in this note are much less conservative than that in [7].

Example 2: Consider the following uncertain discrete-time system with a time-varying delay in the state:

$$
\begin{aligned}
x(k+1)=\left[\begin{array}{cc}
0.8+\alpha(k) & 0 \\
0 & 0.9
\end{array}\right] x(k) & \\
& +\left[\begin{array}{cc}
-0.1 & 0 \\
-0.1 & -0.1
\end{array}\right] x\left(k-d_{k}\right)
\end{aligned}
$$

where $|\alpha(k)| \leq \bar{\alpha}$. The system matrices can be written in the form of (7) with matrices given by

$$
\begin{aligned}
& A_{0}=\left[\begin{array}{cc}
0.8 & 0 \\
0 & 0.9
\end{array}\right] \quad B_{0}=\left[\begin{array}{cc}
-0.1 & 0 \\
-0.1 & -0.1
\end{array}\right] \quad G=\left[\begin{array}{c}
\bar{\alpha} \\
0
\end{array}\right] \\
& H_{1}=\left[\begin{array}{ll}
1 & 0
\end{array}\right] \quad H_{2}=\left[\begin{array}{ll}
0 & 0
\end{array}\right] \quad \Delta(k)=\frac{\alpha(k)}{\bar{\alpha}} .
\end{aligned}
$$

Now assume the time-varying delay $d_{k}$ satisfies $3 \leq d_{k} \leq 5$, and our purpose is to determine the upper value of $\bar{\alpha}$ such that the above system is asymptotically stable. By using Theorem 5 in [7], it is found that the upper value of $\bar{\alpha}$ is 0.1615 , while by using Corollary 2 in this note, the upper value of $\bar{\alpha}$ is found to be 0.2405 , which is much larger. A more detailed comparison is given in Table II. From Table II, we can see that the robust stability condition presented in this note is much less conservative than that in [7]. In particular, when $2 \leq d_{k} \leq 8$ the previous result in [7] is not feasible, while by Corollary 2 we can still conclude that the system is asymptotically stable for $\bar{\alpha} \leq 0.1667$.

Example 3: In this example, we consider an inverted pendulum system with delayed control input. The inverted pendulum on a cart is depicted in Fig. 1. In this system, a pendulum is attached to the side of a cart by means of a pivot which allows the pendulum to swing in

$$
\begin{aligned}
& W=\left[\begin{array}{ccccccccc}
\tau Q_{11}+R_{11} & 0 & 0 & P_{11} & 0 & 0 & \sqrt{d_{M}} M_{1} & \sqrt{d_{M}-d_{m}} S_{1} & \sqrt{d_{M}} N_{1} \\
* & -Q_{11} & 0 & 0 & \epsilon I & 0 & \sqrt{d_{M}} M_{2} & \sqrt{d_{M}-d_{m}} S_{2} & \sqrt{d_{M}} N_{2} \\
* & * & -R_{11} & 0 & 0 & \epsilon I & \sqrt{d_{M}} M_{3} & \sqrt{d_{M}-d_{m}} S_{3} & \sqrt{d_{M}} N_{3} \\
* & * & * & P_{11}+d_{M}\left(Z_{1}+Z_{2}\right) & 0 & 0 & 0 & 0 & 0 \\
* & * & * & * & \epsilon I & 0 & 0 & 0 & 0 \\
* & * & * & * & 0 & \epsilon I & 0 & 0 & 0 \\
* & * & * & * & * & * & -Z_{1} & 0 & 0 \\
* & * & * & * & * & * & * & -Z_{1} & 0 \\
* & * & * & * & * & * & * & * & -Z_{2}
\end{array}\right] \\
& X=\left[\begin{array}{ccccccccc}
M_{1}+N_{1} & S_{1}-M_{1} & -S_{1}-N_{1} & 0 & 0 & 0 & 0 & 0 & 0 \\
M_{2}+N_{2} & S_{2}-M_{2} & -S_{2}-N_{2} & 0 & 0 & 0 & 0 & 0 & 0 \\
M_{3}+N_{3} & S_{3}-M_{3} & -S_{3}-N_{3} & 0 & 0 & 0 & 0 & 0 & 0 \\
0 & 0 & 0 & 0 & 0 & 0 & 0 & 0 & 0 \\
0 & 0 & 0 & 0 & 0 & 0 & 0 & 0 & 0 \\
0 & 0 & 0 & 0 & 0 & 0 & 0 & 0 & 0 \\
0 & 0 & 0 & 0 & 0 & 0 & 0 & 0 & 0 \\
0 & 0 & 0 & 0 & 0 & 0 & 0 & 0 & 0 \\
0 & 0 & 0 & 0 & 0 & 0 & 0 & 0 & 0
\end{array}\right] \\
& U=\left[\begin{array}{llllll}
I & 0 & 0 & 0 & 0 & 0 \\
0 & I & 0 & 0 & 0 & 0 \\
0 & 0 & I & 0 & 0 & 0 \\
0 & 0 & 0 & I & 0 & 0 \\
0 & 0 & 0 & 0 & I & 0 \\
0 & 0 & 0 & 0 & 0 & I \\
0 & 0 & 0 & 0 & 0 & 0 \\
0 & 0 & 0 & 0 & 0 & 0 \\
0 & 0 & 0 & 0 & 0 & 0
\end{array}\right] \\
& V=\left[-(A-I) \quad-B \quad 0 \quad \begin{array}{lllllll}
-(A & 0 & 0 & 0 & 0 & 0
\end{array}\right]
\end{aligned}
$$


TABLE II

CAlculated UpPer VAlues of $\overline{\boldsymbol{\alpha}}$ FOR DifFerent CASES

\begin{tabular}{c|c|c|c|c}
\hline & \multicolumn{4}{|c}{ Maximum value of $\bar{\alpha}$} \\
\hline & $3 \leq d_{k} \leq 5$ & $5 \leq d_{k} \leq 7$ & $2 \leq d_{k} \leq 7$ & $2 \leq d_{k} \leq 8$ \\
\hline By [7] & 0.1615 & 0.1300 & 0.0830 & infeasible \\
\hline By Corollary 2 & 0.2405 & 0.1933 & 0.1901 & 0.1667 \\
\hline
\end{tabular}

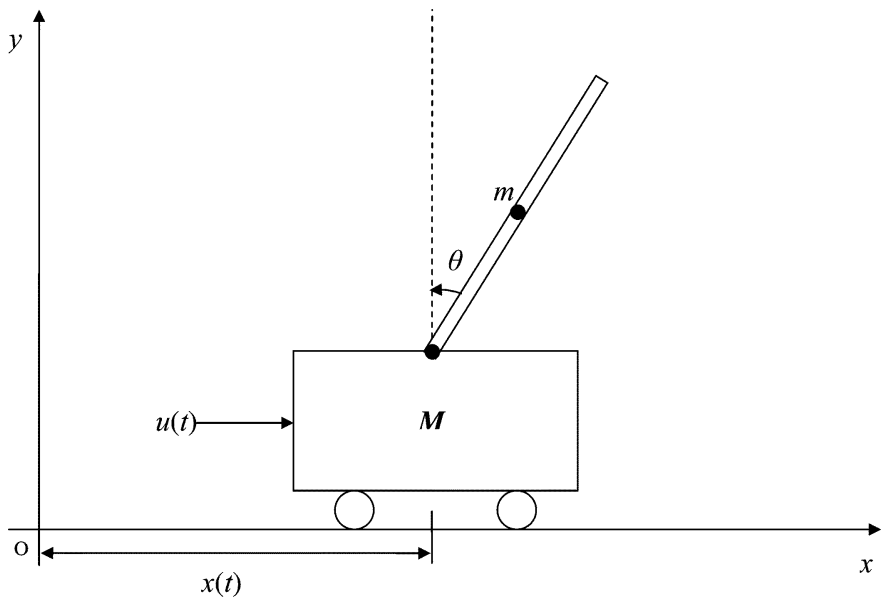

Fig. 1. Inverted pendulum system.

the $x y$-plane. A force $u$ is applied to the cart in the $x$ direction, with the purpose of keeping the pendulum balanced upright. $x$ is the displacement of the center of mass of the cart from the origin $O ; \theta$ is the angle of the pendulum from the top vertical; $M$ and $m$ are the masses of the cart and the pendulum, respectively; $l$ is the half length of the pendulum (i.e., the distance from the pivot to the center of mass of the pendulum). It is assumed that the pendulum is modeled as a thin rod, and the surface is assumed to be friction free. Then, by applying Newton's second law, we arrive at the equations of motion for the system [2]

$$
\begin{aligned}
& (M+m) \ddot{x}+m l \ddot{\theta} \cos \theta-m l \dot{\theta}^{2} \sin \theta=u \\
& m l \ddot{x} \cos \theta+\frac{4}{3} m l^{2} \ddot{\theta}-m g l \sin \theta=0
\end{aligned}
$$

where $g$ is the acceleration due to gravity. Now, by selecting state variables $z=\left[\begin{array}{ll}z_{1} & z_{2}\end{array}\right]^{T}=\left[\begin{array}{ll}\theta & \dot{\theta}\end{array}\right]^{T}$, and by linearizing the above model at the equilibrium point $z=0$, we obtain the following state-space model:

$$
\dot{z}(t)=\left[\begin{array}{cc}
0 & 1 \\
\frac{3(M+m) g}{l(4 M+m)} & 0
\end{array}\right] z(t)+\left[\begin{array}{c}
0 \\
-\frac{3}{l(4 M+m)}
\end{array}\right] u(t) .
$$

Here the parameters are selected as $M=8.0 \mathrm{~kg}, m=2.0 \mathrm{~kg}, l=0.5$ $\mathrm{m}, g=9.8 \mathrm{~m} / \mathrm{s}^{2}$. By assuming the sampling time to be $T_{s}=30 \mathrm{~ms}$, the discretized model for the above pendulum system in (22) is given by

$$
\begin{aligned}
x(k+1)=\left[\begin{array}{ll}
1.0078 & 0.0301 \\
0.5202 & 1.0078
\end{array}\right] x(k) & \\
& +\left[\begin{array}{l}
-0.0001 \\
-0.0053
\end{array}\right] u(k) .
\end{aligned}
$$

The poles of the system are 1.1329 and 0.8827 , thus this discretized system is unstable. It is assumed that a state-feedback delayed control law is given by with $d_{m} \leq d_{k} \leq d_{M}$. When $d_{k}=0$, the closed-loop system has poles placed at 0.9962 and 0.5809 and is thus stable. Now, assume $d_{m}=1$, and we are interested in the upper value of $d_{M}$ such that the closed-loop system remains to be asymptotically stable. By using [7, Th. 1], it is found that the upper delay bound $d_{M}=1$. However, by applying Theorem 1, we obtain the upper delay bound $d_{M}=2$, and by Theorem 3 the upper delay bound $d_{M}=3$, for which the closed-loop system is asymptotically stable. These results show again that the stability conditions presented in this note are generally less conservative.

\section{CONCLUSION}

By defining new Lyapunov functions and by making use of novel techniques to achieve delay dependence, several new results have been obtained for the stability analysis of discrete-time systems with a timevarying delay in the state. The merit of the proposed conditions lies in their less conservativeness, which is achieved by avoiding the utilization of bounding inequalities for cross products between two vectors and by paying careful attention to the subtle difference between the terms $\sum_{m=k-d_{k}}^{k-1}(\bullet)$ and $\sum_{m=k-d_{M}}^{k-1}(\bullet)$. Three examples have been provided to show the advantage of the developed results.

\section{REFERENCES}

[1] M. Basin, J. Perez, and R. Martinez-Zuniga, "Optimal filtering for nonlinear polynomial systems over linear observations with delay," Int. J. Innov. Comput., Inform., Control, vol. 2, pp. 863-874, 2006.

[2] R. H. Cannon, Dynamics of Physical Systems. New York: McGrawHill, 1967.

[3] B. Chen, J. Lam, and S. Xu, "Memory state feedback guaranteed cost control for neutral delay systems," Int. J. Innov. Comput., Inform., Control, vol. 2, pp. 293-303, 2006.

[4] J. Chen, D. M. Xu, and B. Shafai, "On sufficient conditions for stability independent of delay," IEEE Trans. Automat. Control, vol. 40, no. 9, pp. 1675-1680, Sep. 1995.

[5] W. H. Chen, Z. H. Guan, and X. Lu, "Delay-dependent guaranteed cost control for uncertain discrete-time systems with delay," Proc. Inst. Elect. Eng. D: Control Theory Appl., vol. 150, no. 4, pp. 412-416, 2003.

[6] E. Fridman and U. Shaked, "Stability and guaranteed cost control of uncertain discrete delay systems," Int. J. Control, vol. 78, no. 4, pp. 235-246, 2005.

[7] H. Gao, J. Lam, C. Wang, and Y. Wang, "Delay-dependent outputfeedback stabilisation of discrete-time systems with time-varying state delay," Proc. Inst. Elect. Eng. D: Control Theory Appl., vol. 151, pp. 691-698, 2004.

[8] H. Gao and C. Wang, "Comments and further results on "A descriptor system approach to $\boldsymbol{H}_{\infty}$ control of linear time-delay systems"," IEEE Trans. Autom. Control, vol. 48, no. 3, pp. 520-525, Mar. 2003.

[9] K. Gu, V. L. Kharitonov, and J. Chen, Stability of Time-Delay Systems. Berlin, Germany: Springer-Verlag, 2003.

[10] Y. He, Q. G. Wang, C. Lin, and M. Wu, "Delay-range-dependent stability for systems with time-varying delay," Automatica, vol. 43, no. 2, pp. 371-376, 2007

[11] Y. He, M. Wu, J. H. She, and G. P. Liu, "Parameter-dependent Lyapunov functional for stability of time-delay systems with polytopictype uncertainties," IEEE Trans. Autom. Control, vol. 49, no. 5, pp. 828-832, May 2004

[12] C. Lin, Q.-G. Wang, and T. H. Lee, "A less conservative robust stability test for linear uncertain time-delay systems," IEEE Trans. Autom. Control, vol. 51, no. 1, pp. 87-91, Jan. 2006.

[13] H. Liu, F. Sun, K. He, and Z. Sun, "Design of reduced-order $\boldsymbol{H}_{\infty}$ filter for Markovian jumping systems with time delay," IEEE Trans. Circuits Syst. II, vol. 51, no. 11, pp. 607-612, Nov. 2004

[14] Z. Wang, B. Huang, and H. Unbehauen, "Robust $\boldsymbol{H}_{\infty}$ observer design of linear state delayed systems with parametric uncertainty: The discrete-time case," Automatica, vol. 35, pp. 1161-1167, 1999.

[15] Y. Xia and Y. Jia, "Robust stability functionals of state delayed systems with polytopic type uncertainties via parameter-dependent Lyapunov functions," Int. J. Control, vol. 75, pp. 1427-1434, 2002.

[16] L. Xie, "Output feedback $\boldsymbol{H}_{\infty}$ control of systems with parameter uncertainty," Int. J. Control, vol. 63, pp. 741-750, 1996.

$$
u(k)=\left[\begin{array}{ll}
102.9100 & 80.7916
\end{array}\right] x\left(k-d_{k}\right)
$$


[17] S. Xu and J. Lam, "Improved delay-dependent stability criteria for time-delay systems," IEEE Trans. Autom. Control, vol. 50, no. 3, pp. 384-387, Mar. 2005.

[18] S. Xu, J. Lam, and Y. Zou, "Improved conditions for delay-dependent robust stability and stabilization of uncertain discrete time-delay systems," Asian J. Control, vol. 7, no. 3, pp. 344-348, 2005.

\section{Delayed Observers for Linear Systems With Unknown Inputs}

Shreyas Sundaram and Christoforos N. Hadjicostis

\begin{abstract}
We present a method for constructing reduced-order state observers for linear systems with unknown inputs. Our approach provides a characterization of observers with delay, which eases the established necessary conditions for existence of unknown input observers with zero-delay. We develop a parameterization of the observer gain that decouples the unknown inputs from the estimation error, and we use the remaining freedom to ensure stability of the error dynamics. Our procedure is quite general in that it encompasses the design of full-order observers via appropriate choices of design matrices.
\end{abstract}

Index Terms-State estimation, system inversion, unknown input observer.

\section{INTRODUCTION}

In practice, it is often the case that a dynamic system can be modeled as having unknown inputs. For example, in decentralized control, it may not be possible to have knowledge of the control signals generated by different controllers [1]. Unknown inputs can also be used to represent uncertain system dynamics and faults [2]. The problem of constructing an observer for such systems has received considerable attention over the past few decades, and various methods of realizing both full and reduced-order observers have been presented in the literature (e.g., [3]-[5]). In [6], Valcher presented an elegant design procedure to construct full-order observers for linear systems with unknown inputs, along with necessary and sufficient conditions for the existence of such observers. The investigations by Valcher and other researchers have demonstrated that strict conditions must be met in order to reconstruct the entire state vector in the presence of unknown inputs. In [7], it was shown that these conditions can be relaxed by allowing delays in the observer, but no design procedure was provided. In [8], the authors handled delayed observers by constructing a higher dimensional system which incorporated the delayed states into the new state vector. Geometric conditions were then given for the existence of an observer for this augmented system; this approach, however, may cause the dimension of the observer to be much larger than the dimension of the system.

Manuscript received January 9, 2006; revised May 22, 2006 and October 6, 2006. This material is based upon work supported in part by the National Science Foundation (NSF) under NSF Career Award 0092696 and NSF EPNES Award 0224729, and in part by the Air Force Office of Scientific Research (AFOSR) under URI Award F49620-01-1-0365URI. Any opinions, findings, and conclusions or recommendations expressed in this publication are those of the authors and do not necessarily reflect the views of the NSF or the AFOSR.

The authors are with the Coordinated Science Laboratory and the Department of Electrical and Computer Engineering, University of Illinois at Urbana-Champaign, Urbana, IL 61801-2307 USA (e-mail: ssundarm@uiuc.edu; chadjic@uiuc.edu).

Digital Object Identifier 10.1109/TAC.2006.890371
In this note, we provide a design procedure to construct reducedorder observers with delays. Specifically, our approach generalizes the design procedure proposed by Valcher to the case of reduced-order delayed observers, and allows us to treat the full-order observer as a special case of a reduced-order observer where the dynamic portion reconstructs the entire state vector. In contrast to the work in [8], the dimension of our observer is no greater than the dimension of the original system, and we present algebraic existence conditions. Observers that produce delayed estimates of the system state have the potential to be used in a variety of applications, including feedback control [9], chaotic communication systems [10], and fault detection and identification [2]. Of course, in all of these applications, it will be desirable to construct an observer with the smallest possible delay, which is a feature of the observers provided by our design procedure.

\section{UNKNOWN INPUT OBSERVER}

Consider a discrete-time linear system $\mathcal{S}$ of the form

$$
\begin{aligned}
x_{k+1} & =A x_{k}+B u_{k} \\
y_{k} & =C x_{k}+D u_{k}
\end{aligned}
$$

with state vector $x \in \mathbb{R}^{n}$, unknown input $u \in \mathbb{R}^{m}$, output $y \in \mathbb{R}^{p}$, and system matrices $(A, B, C, D)$ of appropriate dimensions. Note that known inputs can be handled in a straightforward manner, and so we omit them for clarity of development. We also assume without loss of generality that the matrix $\left[\begin{array}{l}B \\ D\end{array}\right]$ is full column rank. This assumption can always be enforced by an appropriate transformation and renaming of the unknown input signals.

The response of system (1) over $\alpha+1$ time units $(\alpha=0,1,2, \ldots)$ is given by

$$
\begin{aligned}
\underbrace{\left[\begin{array}{c}
y_{k} \\
y_{k+1} \\
\vdots \\
y_{k+\alpha}
\end{array}\right]}_{Y_{k: k+\alpha}}=\underbrace{\left[\begin{array}{c}
C \\
C A \\
\vdots \\
C A^{\alpha}
\end{array}\right]}_{\Theta_{\alpha}} x_{k} \\
+\underbrace{\left[\begin{array}{cccc}
D & 0 & \cdots & 0 \\
C B & D & \cdots & 0 \\
\vdots & \vdots & \ddots & \vdots \\
C A^{\alpha-1} B & C A^{\alpha-2} B & \cdots & D
\end{array}\right]}_{M_{\alpha}} \underbrace{\left[\begin{array}{c}
u_{k} \\
u_{k+1} \\
\vdots \\
u_{k+\alpha}
\end{array}\right]}_{U_{k: k+\alpha}} .
\end{aligned}
$$

The matrices $\Theta_{\alpha}$ and $M_{\alpha}$ in the previous equation can be expressed in a variety of ways. We will be using the following identities in our derivations:

$$
\begin{aligned}
\Theta_{\alpha} & =\left[\begin{array}{c}
C \\
\Theta_{\alpha-1} A
\end{array}\right]=\left[\begin{array}{c}
\Theta_{\alpha-1} \\
C A^{\alpha}
\end{array}\right] \\
M_{\alpha} & =\left[\begin{array}{cc}
D & 0 \\
\Theta_{\alpha-1} B & M_{\alpha-1}
\end{array}\right]=\left[\begin{array}{ll}
M_{\alpha-1} & 0 \\
C \zeta_{\alpha-1} & D
\end{array}\right]
\end{aligned}
$$

where $\zeta_{\alpha-1} \equiv\left[\begin{array}{llll}A^{\alpha-1} B & A^{\alpha-2} B & \cdots & B\end{array}\right]$.

Definition 1 (Rank-d Linear Functional): Let $\Gamma$ be a $d \times n$ matrix with rank $d$. Then the quantity $\Gamma x_{k}$ will be termed a rank- $d$ linear functional of the state vector $x_{k}$.

We are now ready to proceed with the construction of an observer to estimate the states in $\mathcal{S}$. We start by determining the set of states that can be directly obtained from the output of the system over $\alpha+1$ 